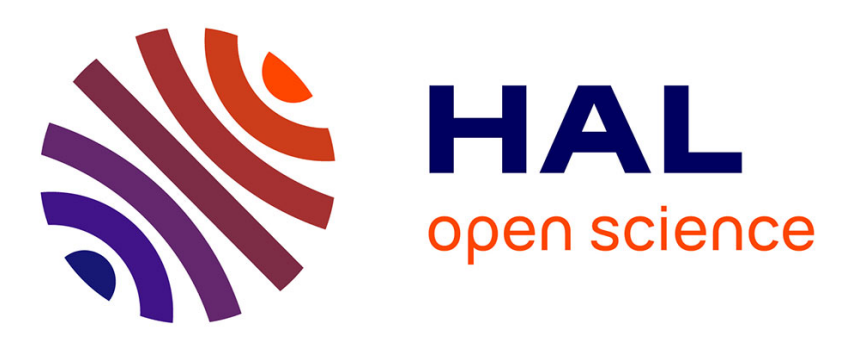

\title{
Modeling and Algorithm on a Class of Mechanical Systems with Unilateral Constraints \\ Feng Qi, J. Tu
}

\section{To cite this version:}

Feng Qi, J. Tu. Modeling and Algorithm on a Class of Mechanical Systems with Unilateral Constraints. Archive of Applied Mechanics, 2006, 76 (1-2), pp.103-116. 10.1007/s00419-006-0008-x . hal-01578898

\section{HAL Id: hal-01578898 \\ https://hal.science/hal-01578898}

Submitted on 30 Aug 2017

HAL is a multi-disciplinary open access archive for the deposit and dissemination of scientific research documents, whether they are published or not. The documents may come from teaching and research institutions in France or abroad, or from public or private research centers.
L'archive ouverte pluridisciplinaire HAL, est destinée au dépôt et à la diffusion de documents scientifiques de niveau recherche, publiés ou non, émanant des établissements d'enseignement et de recherche français ou étrangers, des laboratoires publics ou privés. 


\title{
Q. Feng · J. Tu
}

\section{Modeling and algorithm on a class of mechanical systems with unilateral constraints}

\begin{abstract}
In the present paper, the cable-structures are considered as a class of mechanical complementaryslackness systems. Based on the optimization algorithms used for multi-body dynamics with unilateral contacts, an algorithm by means of artificial neural network (NNW) is developed. The following two classes of cable-structures have been considered: force-elongation of cable member follows elastic behavior and workhardening assumption. Due to simplicity the former is used to prove the method reliability, and the latter, as general cable-structure problem is handled. First, the complementarity problems for those structures have been formulated; then using generalized Gaussian' least action principle they are summarized as an optimization problem. Based on Hopfield's work, an artificial NNW has been designed and used to decide combination of possible constraints at each step in simulation. As examples, two cable-structures have been investigated. The calculated results for a simple suspension structure evidence the reliability and time-economization of the proposed method. An example of guyed mast shows the suitability of the proposed method for practical cable-structures.
\end{abstract}

Keywords cable - structure, artificial NNW, linear complementarity problem

\section{Introduction}

The analysis of cable systems in the geometrically nonlinear range had been the object of a number of studies, for example, Greenberg [1], Jonatowski and Birnstiel [2], Murray and Willems [3] and Tene and Epstein [4]. But the behavior of individual cables becomes highly nonlinear if slackening and plastic tensile strains intervene. This behavior of cable can be called physical nonlinearity. Because of this physical nonlinearity the cable-structure belongs to a class of mechanical systems with unilateral constraint.

To avoid loosening phenomenon of the cables for practical constructions, pre-tension of cables is always used in design. However, for some cable-structures loosening phenomenon occurs still under dynamical load. As a typical example, in 1963, the roof of station hall Villars was damaged under wind load [5]. The model experiment has shown that when an unsymmetrical load acts on this structure, some cable members may be loosening and the roof may be floating. Therefore description with consideration of only geometric nonlinearity for cable-structure can be not realistic. So by modeling structures with cables, the behavior of unilateral constraint must be considered.

The consideration of nonsmooth behavior for the cable-structures may date back to the 1970s. In 1975, Maier et al. [6] had dealt with energy approach to inelastic cable-structure analysis, in which they considered geometric nonlinearity and physical nonlinearity of cable members. They had adopted piecewise linear 
constitutive idealizations, particularly in limit and elastoplastic-work-hardening analysis. Their aim was to alleviate difficulties by using of smooth models [6]. In 1976, Panagitopoulos had dealt with the inelastic, stressunilateral analysis of cable-structures undergoing large displacements [7]. The response of the cable-structures to load- and initial strain-increments is described by a set of equations and inequalities, which are formulated compactly as variational inequalities. Two dual extremum principles with inequalities as subsidiary conditions are derived. The numerical calculation is performed iteratively by using the decomposition techniques of the multilevel optimization and applying to the resulting substructures the algorithms of non-linear optimization. Based on the above mentioned works, analysis of cable-structures may be included in the complementarity problems. But at that time that study was on static or quasi-static problems.

The mechanics with unilateral constraint as an important subclass of non smooth mechanics develops rapidly since 35 years [8]. This field consists of two parts in the area of variational inequalities in connection with convex energy functions [9-12] and the area of hemivariational inequalities in connection with non-convex energy function, which is only 15 years old [13]. Most nonsmooth mechanical problems possess non-convex features. In [11] Panagiotopoulos considered inequality problems, which led him consistently to the development of hemivariational inequalities [13]. His works established the mathematic base of modeling and algorithm on cable-structures.

Nonsmooth mechanics allows a general theoretical description but relevant problems must be solved numerically. In spite of many value contributions to the numerics of non-continuous systems [14] the existing algorithms are still extremely time-consuming. Therefore the numerical solution of all kinds of complementarity problems is a topic of current research. Recently, many researchers are interested in the study of numerical algorithms subjected to unilateral constraints. The significant step may be to decide the real one of possible combination of constraints. For example, by means of common numerical method, if the number of cable members is equal to $m$, one must search for the real one among $2^{m}$ possible combination of constraints and therefore it consumes a large of time on computer. This is not suitable for calculating of a real construction. Also in this paper development of general computer method will be devoted to calculate nonlinear vibrations of cable-structures.

Actually, it has been noticed that non-smooth models overstep the framework of mechanical systems, since they also can apply for instance to electrical and NNW. In the present paper, the cable-structures are considered as a class of mechanical complementary-slackness systems. Based on the optimization algorithms for multi-body dynamics with unilateral contacts, an algorithm by means of artificial NNW has been developed. The following two classes of cable-structures have been considered: force-elongation of cable member follows elastic behavior and work-hardening assumption. Due to simplicity the former is used to prove the method reliability, and the latter as general cable-structure problem is handled. First, the complementarity problems for those structures have been formulated; then using generalized Gaussian' least action principle they are summarized as an optimization problem. Based on Hopfield's work, an artificial NNW has been designed and used to decide combination of possible constraints at each step in simulation. As examples, two cable-structures have been investigated. The calculated results for a simple suspension structure evidence the reliability and time-economization of the proposed method. An example of guyed mast shows the suitability of the proposed method for practical cable-structures.

\section{Modeling of cable-structures as some complementarity problems}

Although our work is aimed at cable-structures, the method proposed here is also available for some hybrid dynamical systems, like convex systems [15, 16], or so-called complementary-slackness systems [17, 18]. As examples in this paper, let us consider the following two systems with different nonlinear behaviors, to model those systems as complementary problems.

It is necessary to have a brief review of cable behaviors. It was concluded that the assumption of elastic behavior (Fig. 1a) of the structure yields the largest tensions in the cables [7]. The elastic-prefectly plastic assumption (Fig. 1b) gives the lowest tensions in the cables. The work-hardening assumption (Fig. 1c) gives tensions between them. It is clear that the developed theory permits us to analyze the calble-structures in their "unstable region" because the possibility that a cable member can become slack is taken into account. In this paper, two cases, elastic behavior and work-hardening assumption, are considered. 


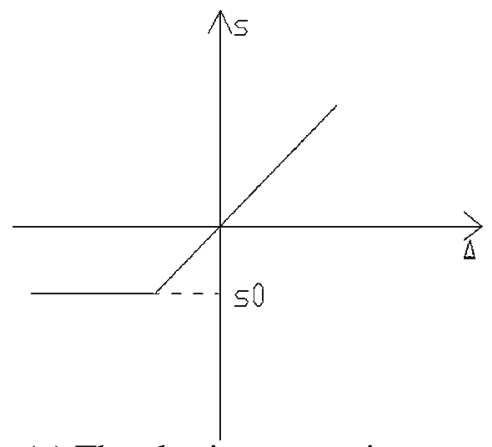

(a) The elastic assumption

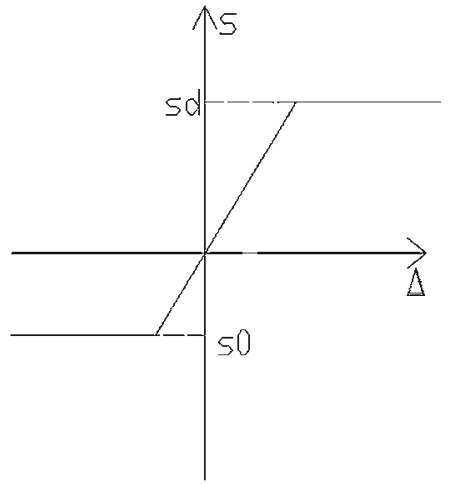

(b) The elastic-prefectly plastic assumption

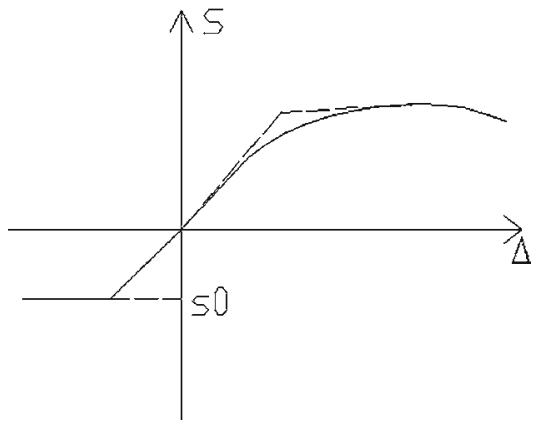

(c) The workhardening assumption

Fig. 1 The elongation-tension curves of cables. a The elastic assumption. b The elastic-prefectly assumption. $\mathbf{c}$ The workhardening assumption

2.1 Modeling of cable-structures as linear complementarity systems

It is well known that cable-structures can be modeled as linear complementarity systems [6,7], but very little work for dynamical problems can be found. In the first example, a simple suspension structure is modeled as a Linear Complementarity Dynamical System.

\subsubsection{Linear complementarity dynamical system}

The governing equations of a Linear Complementarity Dynamical Systems $[8,19,20]$ are written as

$$
\left\{\begin{array}{c}
\dot{x}=A x+B \lambda, \\
y=C x+D \lambda, \\
y(t) \geqslant 0, \lambda(t) \geqslant 0, \quad y(t)^{\mathrm{T}} \lambda(t)=0 .
\end{array}\right.
$$




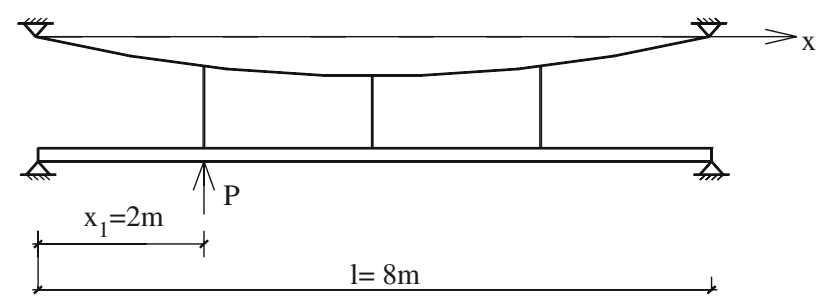

Fig. 2 A simple suspension structure

The modes of this hybrid dynamical system (there are $2^{m}$ modes, y and $\lambda \in R^{m}$ ) correspond to $y_{i}=0$, for $i \in I \subseteq\{1, \ldots, m\}, \quad \lambda_{i}=0$, for $i \in I^{C}$, where $I^{C}$ is the complement of $\mathrm{I}$ in $\{1, \ldots, m\}$. It is assumed that each mode is autonomous, which means that each consistent state $x_{0}$ there corresponds a unique cou$\operatorname{ple}(x(t), \lambda(t))$, with $\lambda(\bullet)$ smooth.

If cable-elements are elastic, see Fig. 1a, some cable-structures can be described by Eqs. (1). To prove the reliability of the method proposed in this paper, a simple example has been considered firstly in the following subsection.

\subsubsection{A simple example for a suspension structure}

A simple suspension structure showing in Fig. 2 consists of a main cable, three slings and a beam. An unsymmetrical external force $p(t)$ acts at 1/4 position of the beam. Each sling is assumed to be elastic, see Fig. 1a.

Let the axial elongation of the $\mathrm{i}$-th suspension sling be

$$
\Delta l_{i}=\Delta l_{i 0}-w_{i}-u_{i}(i=1,2,3),
$$

where $\Delta l_{i 0}$ - static expansion of the i-th sling;

$w_{i}$ - elastic deflection of the beam at connected point with the $i$-th sling;

$u_{i}$ - elastic deflection of the main cable at connected point with the $i$-th sling;

The governing equations of the entire system are given as follows:

$$
\left\{\begin{array}{c}
m_{C} \ddot{u}-H u^{\prime \prime}=\sum_{i=1}^{s} F_{i} \delta\left(x-x_{i}\right), \\
m_{B} \frac{\partial^{2} w}{\partial t^{2}}+\mathrm{EI} \frac{\partial^{4} w}{\partial x^{4}}+\mathrm{CI} \frac{\partial^{5} w}{\partial t \partial x^{4}}=\sum_{i=1}^{s} F_{i} \delta\left(x-x_{i}\right)+P(t),
\end{array}\right.
$$

where $m_{c}$ and $m_{B}$ are mass permit unit length of the cable and the beam respectively. $E$ is the Young's modulus of the beam; $C$ indicates the damping factor; and $P(t)$ describes the external excitation; $F_{i}$ is the force of the $i$-th sling acting at beam and main cable.

The boundary condition can be expressed by

$$
\begin{aligned}
u(0, t) & =u(l, t)=0 ; \\
w(0, t) & =w(l, t)=\left.\frac{\partial^{2} w}{\partial x^{2}}\right|_{x=0}=\left.\frac{\partial^{2} w}{\partial x^{2}}\right|_{x=l}=0 .
\end{aligned}
$$

Introducing the following relations

$$
u=\sum_{n=1}^{\infty} \psi_{2 n}(x) v_{2 n}(t) ; \quad w=\sum_{j=1}^{\infty} \varphi_{j}(x) q_{j}(t) .
$$

Take into account the boundary conditions, one has

$$
\phi_{j}(x)=\sin \frac{j \pi}{l} x \quad(\mathrm{j}=1,2,3 \ldots) ; \quad \psi_{2 n}(x)=\sin \frac{2 n \pi}{l} x \quad(n=1,2,3, \ldots) .
$$


Substituting expression (4) into Eq. (3), and integrating the resulting equations, the equations of motion for the entire structure can be rewritten as

$$
\left\{\begin{array}{l}
\ddot{q}_{j}+2 \xi_{B j} \omega_{B j} \dot{q}_{j}+\omega_{B j}^{2} q_{j}=\sum_{i=1}^{3} \frac{\phi_{j}\left(x_{i}\right)}{M_{B j}} F_{s i}+\frac{P_{j}}{M_{B j}}, \\
\ddot{v}_{2 n}+\omega_{C 2 n}^{2} v_{2 n}=\sum_{i=1}^{3} \frac{\psi_{2 n}\left(x_{i}\right)}{M_{C 2 n}} F_{S i},
\end{array}\right.
$$

where

$$
\begin{aligned}
M_{B_{j}} & =m_{B} \int_{0}^{l} \phi_{j}^{2}(x) \mathrm{d} x, \quad \omega_{B_{j}}=\left(\frac{j \pi}{l}\right)^{2} \sqrt{\frac{\mathrm{EI}}{m_{B}}}, \quad \xi_{B_{j}}=\frac{C \omega_{B_{j}}}{2 E}, \quad P_{j}=\int_{0}^{l} \phi_{j}(x) P \mathrm{~d} x ; \\
M_{C_{2 n}} & =m_{C} \int_{0}^{l} \psi_{2 n}^{2}(x) \mathrm{d} x, \quad \omega_{C_{2 n}}=\frac{2 n \pi}{l} \sqrt{\frac{H}{m_{C}}} .
\end{aligned}
$$

Let $Y=\left[Y_{q}, Y_{v}\right]^{\mathrm{T}}=\left[q_{1} q_{2} \ldots q_{j} v_{21} v_{22} \ldots v_{2 n}\right]^{\mathrm{T}}$, the above equations can be rewritten in the following form:

$$
\left\{\begin{array}{l}
\ddot{Y}+\Xi \dot{Y}+\Omega Y=F_{K} \Delta S+F_{P}=F_{K} K|\Delta L| \lambda+F_{P} \\
\Delta S=K \Delta L=K\left(L-L_{0}\right)=K D Y \geqslant 0
\end{array}\right.
$$

in which,

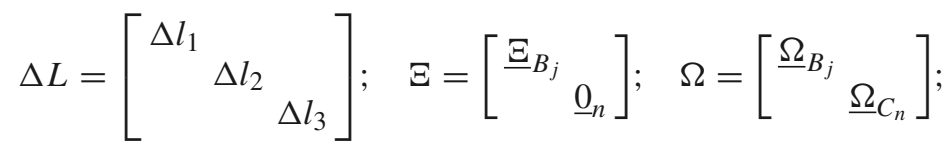

$$
\begin{aligned}
& D=\left[\varphi_{j} \psi_{j}\right] ; \quad F_{K}=\left[\begin{array}{ccc}
\frac{\varphi_{1}\left(x_{1}\right)}{M_{B_{1}}} & \frac{\varphi_{1}\left(x_{2}\right)}{M_{B_{1}}} & \frac{\varphi_{1}\left(x_{3}\right)}{M_{B_{1}}} \\
\vdots & \ddots & \vdots \\
\frac{\varphi_{j}\left(x_{1}\right)}{M_{B_{j}}} & \frac{\varphi_{j}\left(x_{2}\right)}{M_{B_{j}}} & \frac{\varphi_{j}\left(x_{3}\right)}{M_{B_{j}}} \\
\frac{\psi_{21}\left(x_{1}\right)}{M_{C_{21}}} & \frac{\psi_{21}\left(x_{2}\right)}{M_{C_{21}}} & \frac{\psi_{21}\left(x_{3}\right)}{M_{C_{21}}} \\
\vdots & \ddots & \vdots \\
\frac{\psi_{2 n}\left(x_{1}\right)}{M_{C_{2 n}}} & \frac{\psi_{2 n}\left(x_{2}\right)}{M_{C_{2 n}}} & \frac{\psi_{2 n}\left(x_{3}\right)}{M_{C_{2 n}}}
\end{array}\right] ; \quad F_{P}=\left[\begin{array}{c}
\frac{P_{1}}{M_{B_{1}}} \\
\vdots \\
P_{j} \\
M_{B_{j}} \\
\overline{0_{n}}
\end{array}\right] ; \\
& K=\left[\begin{array}{ccc}
k_{1} & 0 & 0 \\
0 & k_{2} & 0 \\
0 & 0 & k_{3}
\end{array}\right] ; \quad S=|S| \lambda ; \quad \lambda=\left\{\begin{array}{l}
\lambda_{1} \\
\lambda_{2} \\
\lambda_{3}
\end{array}\right\} ; \quad|S|=\left[\begin{array}{ccc}
\left|S_{1}\right| & 0 & 0 \\
0 & \left|S_{2}\right| & 0 \\
0 & 0 & \left|S_{3}\right|
\end{array}\right],
\end{aligned}
$$

where $\lambda_{i}$ indicates as an indicative function of the $i$-th cable tension. According to the elastic tension-elongation relation, tension increment of each sling can be described in the following form.

$$
\Delta S_{i}=k_{i} \Delta l_{i} .
$$

Let $y_{i}=\Delta s_{i}-k_{i} \Delta l_{i}$ and introducing the state vector, $x=\left[\begin{array}{ll}Y & \dot{Y}\end{array}\right]^{\mathrm{T}}$, the mathematical problem (7) can be reformed into a standard Linear Complementarity Dynamical System as described in Eq. (2), in the following form.

$$
\left\{\begin{array}{l}
\dot{x}=A x+\hat{F}_{P}+\hat{F}_{K} \Delta S, \quad y=\Delta S-K \Delta L \\
\Delta S \geqslant 0, \quad y \geqslant 0, \quad \Delta S^{\mathrm{T}} y=0
\end{array}\right.
$$

where

$$
A=\left[\begin{array}{ll}
0 & I \\
-\Omega & -\Xi
\end{array}\right] ; \quad \hat{F}_{P}=\left\{\begin{array}{c}
0 \\
F_{P}
\end{array}\right\} ; \quad \hat{F}_{K}=\left\{\begin{array}{c}
0 \\
F_{K}
\end{array}\right\} .
$$


In Eqs. (9), one can see that

$$
\begin{gathered}
\text { if } \Delta S_{i} \geqslant 0, \quad y_{i}=0, \\
\text { If } \Delta S_{i}=0, \quad \Delta l_{i}<0, \quad y_{i}>0 .
\end{gathered}
$$

2.2 Modeling of cable-structures as a Mechanical Complementary-Slackness System

\subsubsection{Formulation of Complementarity-Slackness Systems}

If cables behave as the work-haderning, the cable-structures may be modeled as a class of ComplementaritySlackness Systems. Let us consider the second example, a guyed mast as a Complementary-Slackness System. This class of systems war defined in [17, 18], namely

$$
\left\{\begin{array}{c}
\dot{x}=f(x, u), \quad y=h(x, u), \\
y \geqslant 0, \quad u \geqslant 0, \quad y^{T} u=0 .
\end{array}\right.
$$

\subsubsection{Example 2: a guyed mast}

Petersen had investigated a guyed mast [19], which is described by DAE. In this mast the space chaotic vibration subjected to wind was discovered. But he did not consider the loosing phenomenon of cables, which may occur under the dynamic wind load. In this paper, based on Petersen's model a new model will be suggested as a Complementarity-Slackness System.

(1) Petersen's model

The equations of space vibration of a guyed mast are given as follows [19]:

$$
\left\{\begin{array}{l}
m \ddot{w}_{x}+d_{1} \dot{w}_{x}+d_{2} \dot{w}_{x}^{2}+R_{x}=F_{x}(t), \\
m \ddot{w}_{y}+d_{1} \dot{w}_{y}+d_{2} \dot{w}_{y}^{2}+R_{y}=F_{y}(t),
\end{array}\right.
$$

where $m$ is effective mass, $d_{1}$ and $d_{2}$ are damping factors, $R_{x}$ and $R_{y}$ indicate the components of restoring forces in the $x$ - and the $y$-directions; $w_{x}$ and $w_{y}$ describe the components of displacements at mast top in the $x$-and the $y$-directions; $F_{x}$ and $F_{y}$ are the components of wind load in the $x$-and the $y$-directions, respectively. Figure 3 shows the structure diagram of a simple guyed mast in which the $x$ indicates the

(a)

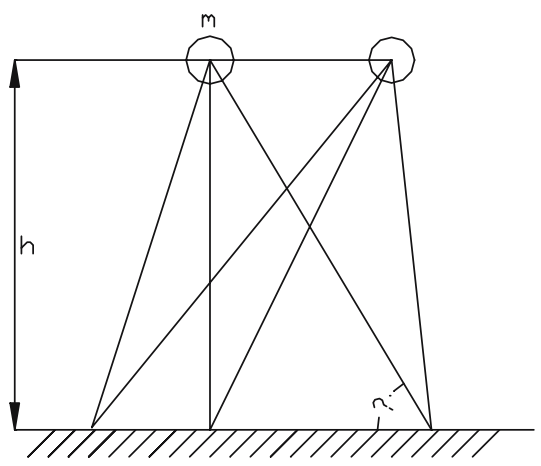

(b)

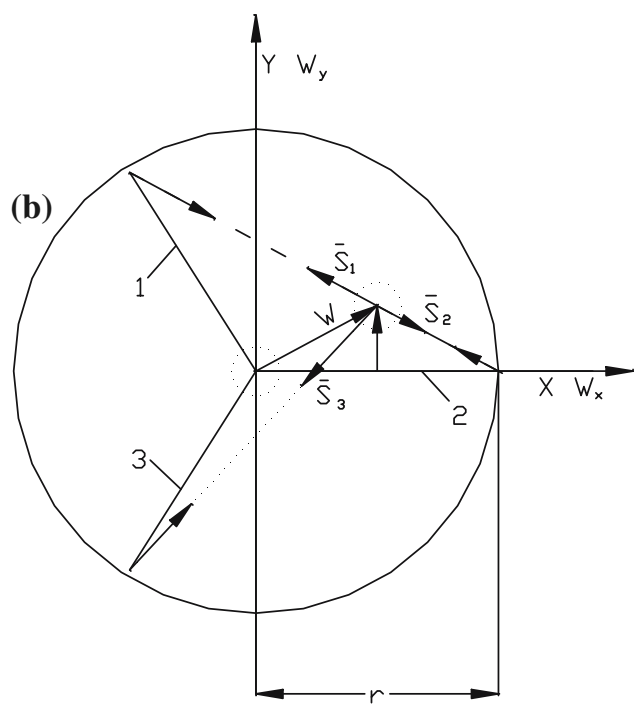

Fig. 3 The structure of a guyed mast 
direction of symmetric area on this mast, and $r$ is distance from the mast to anchoring points. Introducing the dimensionless variables $\xi$ and $\eta$, and let

$$
\xi=w_{x} / r, \quad \text { and } \eta=w_{y} / r
$$

the Eqs. (11) can be rewritten in the following dimensionless form:

$$
\left\{\begin{array}{c}
\ddot{\xi}+\frac{d_{1}}{m} \dot{\xi}+\frac{d_{2} r}{m} \dot{\xi}^{2}+\left(\bar{S}_{1} \cos \bar{\alpha}-\bar{S}_{2} \cos \bar{\beta}+\bar{S}_{3} \cos \bar{\gamma}\right) / r m=F_{x}(t) / r m, \\
\ddot{\eta}+\frac{d_{1}}{m} \dot{\eta}+\frac{d_{2} r}{m} \dot{\eta}^{2}+\left(-\bar{S}_{1} \sin \bar{\alpha}+\bar{S}_{2} \sin \bar{\beta}+\bar{S}_{3} \sin \bar{\gamma}\right) / r m=F_{y}(t) / r m,
\end{array}\right.
$$

in which,

$$
\bar{S}_{i}=S_{i} \cos \delta, \quad i=1,2,3,
$$

where $\bar{S}_{i}$ is the horizontal projection of tension $S_{i}$ of the $i$-th cable, $\delta$ is defined as an inclined angle between the cable and the horizontal plan, and tension $S_{i}$ is the solution of the following algebraic equation:

$$
\left(\frac{S_{i}}{S_{0}}\right)^{3}+c_{i}\left(\frac{S_{i}}{S_{0}}\right)^{2}-a=0
$$

in the above equation, the parameters are given by

$$
\begin{gathered}
a=\frac{g^{2} s^{2} \cos ^{2} \delta}{24 S_{0} E_{s} A_{s}}, \\
b_{i}=\frac{E_{s} A_{s} \cos \delta r}{S_{0} s}, \\
c_{i}=a-b_{i} \breve{\Delta}_{i}-1 ; \quad \breve{\Delta}_{i}=\frac{\Delta_{i}}{r},
\end{gathered}
$$

where $g$ is the weight per unit length of the cable; $s$ means the length of the cable; $E_{s}$ stands for the Young's modulus; $A_{s}$ is cross-sectional area of the cable; $S_{0}$ describes pre-tensile force; $\Delta_{i}$ indicates the elongation of the $i$-th cable and its the first order approximations are given as follows:

$$
\begin{aligned}
& \Delta_{1}=\left(\sqrt{1+\xi-\sqrt{3} \eta+\xi^{2}+\eta^{2}}-1\right) r, \\
& \Delta_{2}=\left(\sqrt{1-2 \xi+\xi^{2}+\eta^{2}}-1\right) r, \\
& \Delta_{3}=\left(\sqrt{1+\xi+\sqrt{3 \eta}+\xi^{2}+\eta^{2}}-1\right) r,
\end{aligned}
$$

where $\breve{\Delta}_{i}$ indicates dimensionless elongation of the $i$-th cable. In Eq. (12), $\bar{\alpha}, \bar{\beta}, \bar{\gamma}$ are defined as inclined angles between the three cables and the $x$-direction, respectively, which can be determined using the following relations.

$$
\begin{aligned}
& \bar{\alpha}=60^{\circ}-\arccos \left[\frac{2+\xi-\sqrt{3} \eta}{2 \sqrt{1+\xi-\sqrt{3} \eta+\xi^{2}+\eta^{2}}}\right], \\
& \bar{\beta}=\arccos \left[\frac{1-\xi}{\sqrt{1-2 \xi+\xi^{2}+\eta^{2}}}\right], \\
& \bar{\gamma}=60^{\circ}-\arccos \left[\frac{2+\xi+\sqrt{3} \eta}{2 \sqrt{1+\xi+\sqrt{3} \eta+\xi^{2}+\eta^{2}}}\right] .
\end{aligned}
$$


(2) A new model as a Complementarity-Snackness System

Some cables may loosen under a largest wind load. A new model is proposed here. It includes the nonsmooth behavior of the structure. Setting $q=[\xi \eta]^{\mathrm{T}}$ and writing the Eq. (14) as $S_{i}=G(\Delta)$, Eqs. (12) may be rewritten in the following form,

$$
\left\{\begin{array}{c}
\ddot{q}+h(\dot{q})-Q(t)+\Gamma S=\ddot{q}+h(\dot{q})-Q(t)+\Gamma|S| \lambda=0, \\
\Delta S=G(\Delta)-S_{0} \geqslant 0,
\end{array}\right.
$$

in which, $\lambda_{i}$ is the indicative function of the $i$-th cable, and where

$$
\begin{aligned}
h(\dot{q}) & =\left[\begin{array}{c}
\frac{d_{1}}{m} \dot{q}_{1}+\frac{d_{2} r}{m} \dot{q}_{1}^{2} \\
\frac{d_{1}}{m} \dot{q}_{2}+\frac{d_{2} r}{m} \dot{q}_{2}^{2}
\end{array}\right] ; \quad Q(t)=\left\{\begin{array}{c}
\frac{F_{x}}{r m} \\
\frac{F_{y}}{r m}
\end{array}\right\} ; \quad \Gamma=\left[\begin{array}{ccc}
\cos \bar{\alpha} & -\cos \bar{\beta} & \cos \bar{\gamma} \\
-\sin \bar{\alpha} & \sin \bar{\beta} & \sin \bar{\gamma}
\end{array}\right] ; \\
S & =\left[\begin{array}{ccc}
\bar{S}_{1} & 0 & 0 \\
0 & \bar{S}_{2} & 0 \\
0 & 0 & \bar{S}_{3}
\end{array}\right] ; \quad \lambda=\left[\begin{array}{l}
\lambda_{1} \\
\lambda_{2} \\
\lambda_{3}
\end{array}\right] .
\end{aligned}
$$

From the Eq. (14), the relation $S=G(\Delta)$ can be derived.

Equation (17) can be rewritten as a standard form of a Complementarity-Snackness System described by Eq. (10). Let $x=\left[\begin{array}{ll}q & \dot{q}\end{array}\right]^{\mathrm{T}}$ and $y=S-G(\Delta)$, one has the following equations:

$$
\left\{\begin{array}{c}
\dot{x}=f(x, S), \quad y=S-G(\Delta(x)) \\
y \geqslant 0, \quad S \geqslant 0, \quad y^{T} S=0
\end{array}\right.
$$

In the Eq. (18), it is clear that

$$
\text { if } S \geqslant 0, \quad y=0 \text { and if } S=0, \Delta \leqslant 0, y>0 .
$$

Summing Eqs. (9) and (18), a general model for cable-structures may be expressed in the following form.

$$
\left\{\begin{array}{c}
\ddot{q}+g(q, \dot{q})=H(q, \dot{q}) S+P(t), \quad y=S-h(q) \\
S \geqslant 0, \quad y \geqslant 0, \quad S^{T} y=0 .
\end{array}\right.
$$

According to Eq. (10), the above problem can belong to Compelementarity-Slackness Systems. It is well known that this problem can be solved using the optimization techniques. But some algorithms of optimization are still CPU-time consuming to decide possible constraints. Hence, a new algorithm of artificial NNW will be proposed in the following section.

\section{The artificial NNW algorithm}

Study of numerical algorithm to systems subjected to unilateral constraints is our main assignment. As a powerful tool by investigation of the dynamic systems with unilateral constraints, the optimization method has been commonly used to solve linear compelementarity problems (LCP) [20]. Using generalized Gaussian' least action principle the Complementarity-Slackness Problem (19) will be summarized as an optimization one.

It is generally agreed that the optimization problem can be solved by means of artificial NNW. The early work for analysis of LCP using the artificial NNW algorithm to solving LCP had been reported in [21]. A new NNW based on a reformation of the LCP into unconstrained minimization problem was proposed. In this paper, based on Hopfield's work a class of artificial NNW will be designed and used to decide the combination of possible constraints at each step of simulation. 


\subsection{The generalized Gaussian's least action principle}

The Gauss's least action principle is one of the several variational principles in mechanics. In fact, Gauss's principle is a reinterpretation of d'Alember's principle into a minimum principle. It turns out that Gauss's principle is also valid for systems subjected to unilateral constraints [22-25]. It is found that integrating of the system at a given time instant is a quadratic procedure, and is closely related to complementarity conditions. The general form of the complementarity conditions can be found in the works by Loetsted [23], Baraff [26] and Moreau [24]. From the above dynamics one may see that a solution to the LCP (19) is equivalent to the following quadratic programming problem (20), and vice-versa.

$$
\begin{aligned}
\min _{\lambda \geqslant 0} G(\lambda)=\frac{1}{2} \lambda^{\mathrm{T}} \nabla_{q} f(q)^{\mathrm{T}} H(q, \dot{q})|S| \lambda+\lambda^{\mathrm{T}}\left[\nabla_{q} f(q)^{\mathrm{T}} z+\frac{\mathrm{d}}{\mathrm{d} t}\left(\nabla_{q} f(q)\right)^{\mathrm{T}} \dot{q}\right] \\
z=-g(q, \dot{q})+P(t), \\
\frac{\partial G}{\partial \lambda}=\nabla_{q} f(q)^{\mathrm{T}} H(q, \dot{q})|S| \lambda+\nabla_{q} f(q)^{\mathrm{T}} z+\frac{\mathrm{d}}{\mathrm{d} t}\left(\nabla_{q} f(q)\right)^{\mathrm{T}} \dot{q} \\
=\nabla_{q} f(q)^{\mathrm{T}}[H(q, \dot{q})|S| \lambda-g(q, \dot{q})+P(t)]+\frac{\mathrm{d}}{\mathrm{d} t}\left(\nabla_{q} f(q)\right)^{\mathrm{T}} \dot{q} \\
=\nabla_{q} f(q)^{\mathrm{T}} \ddot{q}+\frac{\mathrm{d}}{\mathrm{d} t}\left(\nabla_{q} f(q)\right)^{\mathrm{T}} \dot{q}={\frac{\mathrm{d}^{2} f(q)}{\mathrm{d} t^{2}}=0 .}^{T}=0
\end{aligned}
$$

From this, the following dynamical equation can be obtained.

$$
\left\{\begin{array}{c}
\ddot{q}+g(q, \dot{q})=H(q, \dot{q}) S+P(t)=H(q, \dot{q})|S| \lambda+P(t), \\
S=f(q) \geqslant 0,
\end{array}\right.
$$

where the inequalities $S=f(q) \geqslant 0$ can be rewritten into the following complementarity conditions:

$$
y=S-f(q) \geqslant 0 ; \quad S \geqslant 0 ; \quad S^{T} y=0 .
$$

Thus it is proved that the optimization problem (20) and the dynamics with unilateral constraints (19) are equivalent to each other.

\subsection{An algorithm of artificial NNWs}

Much of the resurgence of interest in NNWs during the early 1980s can be attributed to the work of John Hopfield [27]. He wrote two highly influential papers in 1982 [28] and 1984 [29], respectively. A number of important ideas and a clear mathematical analysis in his early papers include content-addressable memory, analog-to-digital conversion, and optimization [30, 31].

The Hopfield network does not have a learning law associated with it. It is not trained, nor does it learn on its own. Instead, a design procedure based on the Lyapunov function is used to determine the weight matrix and the bias vector. This trick, of course, is of convenience.

In this section, adopting Hopfield's method [30], an artificial NNWs equivalent to optimization problem (20) will be designed. Let $V_{i}=\lambda_{i}, V_{i}=F\left(x_{i}\right)$, from the Gaussian's function, an energy function may be assumed to be

$$
\begin{aligned}
E(V)= & \frac{1}{2} V^{\mathrm{T}} \nabla_{q} f(q)^{\mathrm{T}} H(q, \dot{q})|S| V+V^{\mathrm{T}}\left(\nabla_{q} f(q)^{\mathrm{T}} z\right. \\
& \left.+\frac{\mathrm{d}}{\mathrm{d} t}\left(\nabla_{q} f(q)\right)^{\mathrm{T}} \dot{q}\right)+\sum_{i=1}^{m} \frac{1}{R_{i}} \int_{0}^{V_{i}} F(\eta) \mathrm{d} \mu .
\end{aligned}
$$


According to Hopfield's method, we put $\partial E / \partial V_{i}=-\mathrm{d} x_{i} / \mathrm{d} t$. When $t \rightarrow \infty$, and $\mathrm{d} x_{i} / \mathrm{d} t=0$, a stable solution can be obtained as

$$
\left\{\begin{array}{c}
X_{j+1}=W_{j} V_{j}+I_{j}, \\
V_{j+1}=F\left(X_{j}\right)=\left\{\begin{array}{ll}
0, & X_{j} \leqslant 0 \\
1, & X_{j}>0
\end{array},\right.
\end{array}\right.
$$

in which,

$$
\begin{aligned}
W_{j} & =-\nabla_{q} f\left(q_{j}\right)^{\mathrm{T}} H\left(q_{j}, \dot{q}_{j}\right)|S|, \\
I_{j} & =-\left[\nabla_{q} f\left(q_{j}\right)^{\mathrm{T}} z_{j}+\frac{\mathrm{d}}{\mathrm{d} t}\left(\nabla_{q} f\left(q_{j}\right)\right)^{\mathrm{T}} \dot{q}_{j}\right] \\
& =-\left[\nabla_{q} f\left(q_{j}\right)^{\mathrm{T}}\left(-g\left(q_{j}, \dot{q}_{j}\right)+P_{j}\right)+\frac{\mathrm{d}}{\mathrm{d} t}\left(\nabla_{q} f\left(q_{j}\right)\right)^{\mathrm{T}} \dot{q}_{j}\right] .
\end{aligned}
$$

Using artificial NNW to decide the real involved constraints and by means of a modified Rung-Kutta method, the solution of Eqs. (21) can be obtained. The flow chart for this method is shown in Fig. 4.

The description of the flow chart:

(1) Using Runge-kutta method, response of the whole structure given by $(19$, a) at the $j-1$ - step can be obtained;

(2) Introducing the above results into equation of artificial NNW (23), the values $\lambda$ at the $j$-th step can be determined and the involved constraint combination at the next step can be decided;

(3) Substituting the values $\lambda$ at the $j$-th step into the Eq. (19, a) and return to Eq. (23).

\section{Numerical examples and discussion}

\subsection{Example 1: a simple suspension structure}

Figure 2 shows a simple suspension structure, which consists of a main cable, three slings and a beam. The structure parameters are assumed as listed below: length of beam $l=8 \mathrm{~m}$, bending stiffness EI $=1.2 \times 10^{7} \mathrm{~N} / \mathrm{m}^{2}$, mass per unit length of beam $m_{b}=650 \mathrm{~kg} / \mathrm{m}$, stiffness of the sling $k=3.75 \times 10^{3} \mathrm{~N} / \mathrm{m}$, the three slings are arrange at $l / 4, l / 2,3 l / 4$ of beam; mass per unit length of the main cable $m_{c}=100 \mathrm{~kg} / \mathrm{m}$, the droop $f=0.1 \mathrm{~m}$; the external excitation $P=P_{0} \sin (\theta t)$ with $P_{0}=5,000 \mathrm{~N}, \theta=0.75 \omega_{B_{1}}$, acting at $l / 4$ of the beam.

Figures 5 and 6 exhibit the time histories of the deformations and tensions for the three slings using both methods of artificial NNW and general search method (GSM), respectively. In those figures, the results calculated by the both methods are the same. This testifies that the method of artificial NNW is reliable. Moreover, the calculated results show that using the NNW method to solve this simple problem requires only $15 \mathrm{~min}$ of CPU-time, but using GSM needs several hours. From the above mentions one can conclude that it may be better, to use the artificial NNW by solving the dynamics of the system with unilateral constraints.

\subsection{Example 2: a guyed mast}

The structure parameters are the same as Petersen's mode [19],i.e., $\mathrm{s}=240 \mathrm{~m}, \mathrm{~m}=27,500 \mathrm{~kg}, \mathrm{r}=145 \mathrm{~m}$, $\mathrm{g}=85 \mathrm{~N} / \mathrm{m}, A_{s}=1.005 \times 10^{-3} \mathrm{~m}^{2}, E_{s}=1.6 \times 10^{11} \mathrm{~N} / \mathrm{m}^{2}, S_{0}=50,249 \mathrm{~N}, d_{1}=1,000, d_{2}=0, \xi_{0}=0.015$,

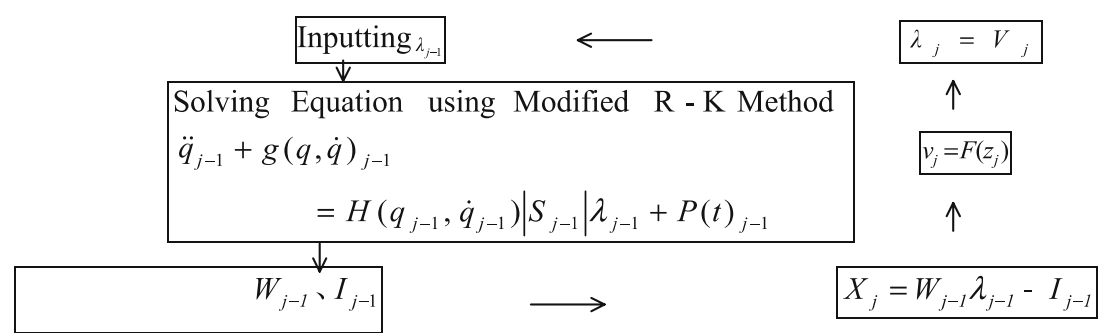

Fig. 4 The flow chart for the method of artificial neural network 


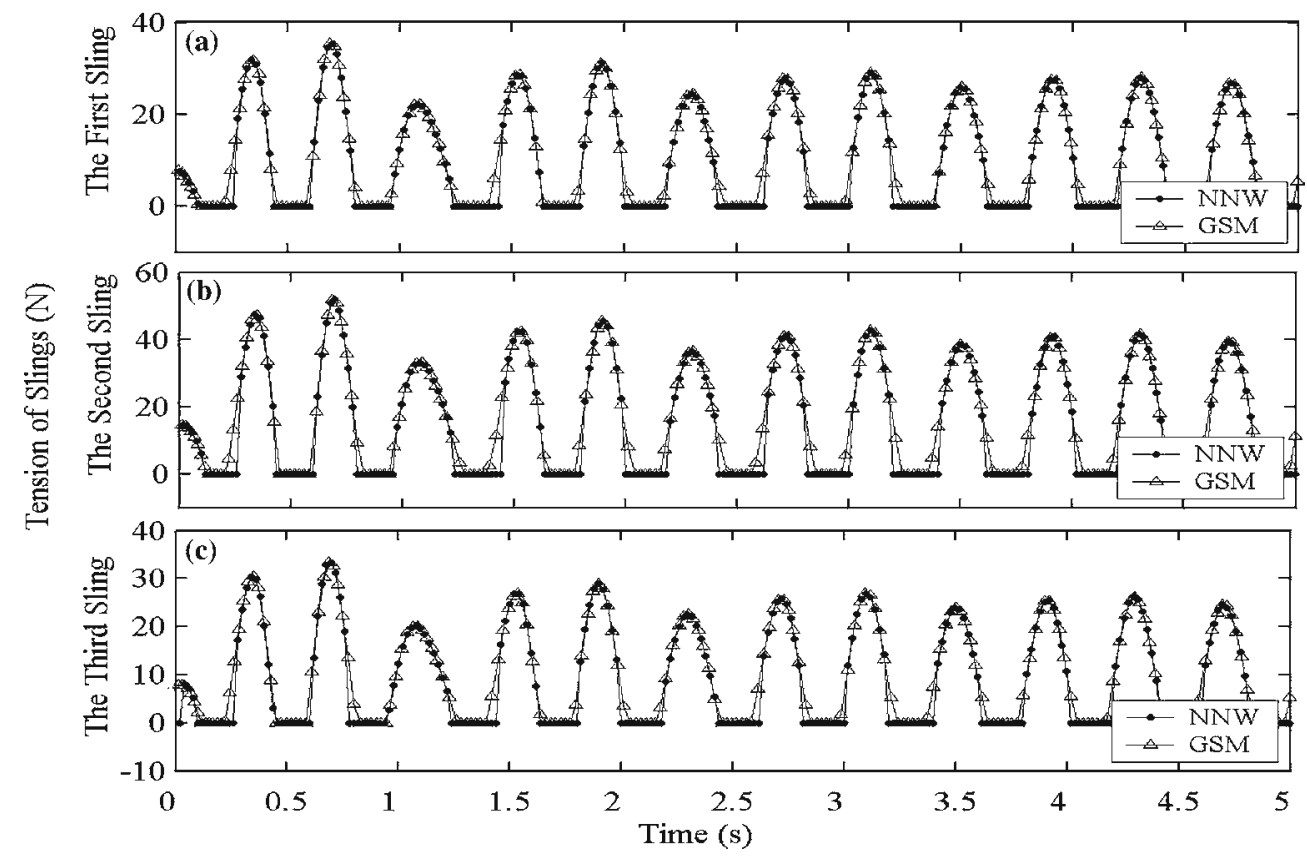

Fig. 5 The time histories of the tensions of the slings

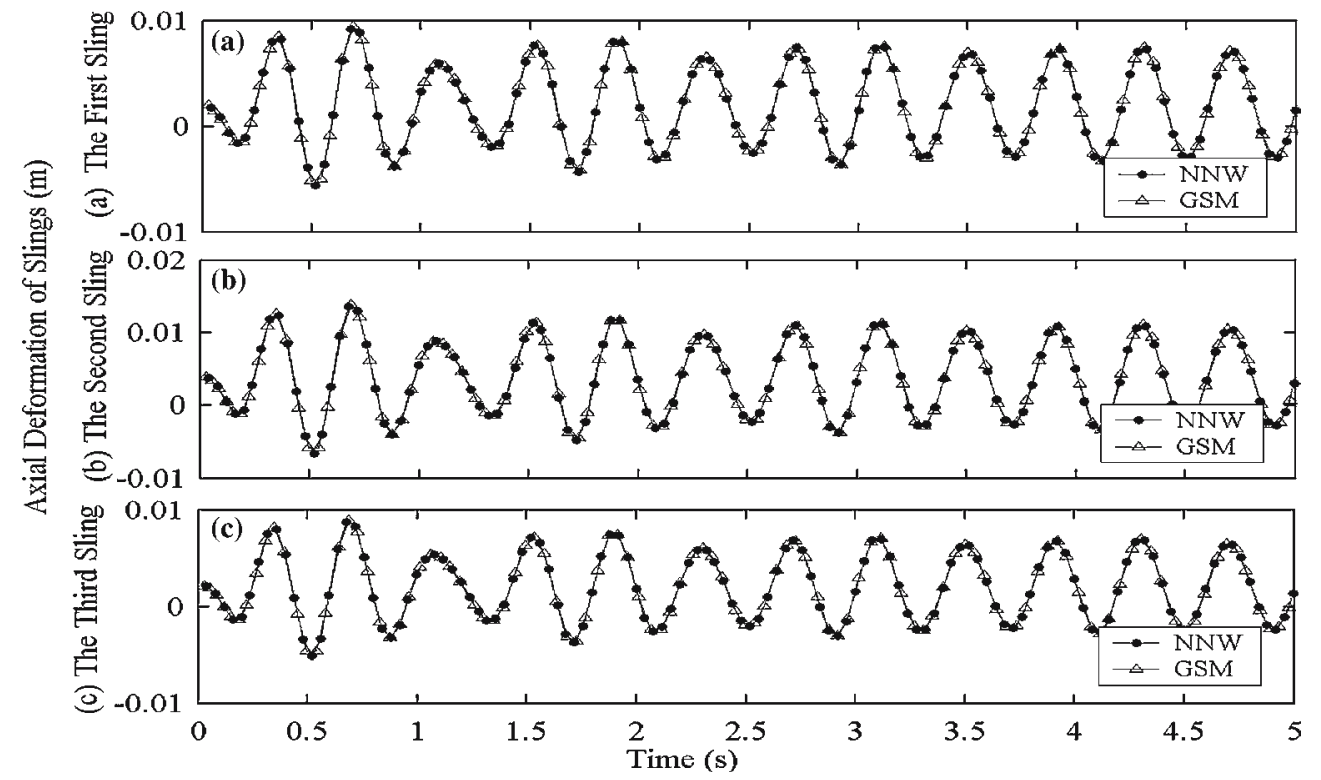

Fig. 6 The time histories of the deformations of the slings

$\eta_{0}=0.0075, f_{x}=33,541 \times \cos \alpha N, f_{y}=33,541 \times \sin \alpha N, \alpha=\pi / 3$ and step $\Delta t=0.1 \mathrm{~s}$, calculated number $N=10,000$.

Figures 7 and Fig. 8 give the results by using Petersen's model and by new model suggested in this paper, respectively. In Fig. 7, one can find negative values of tension, this is not reasonable, while this phenomenon is avoided in the modified model. In Fig. 8, some cables sometime may be loosening, and their tensions are equal to zero. It may be interpreted, that for this example the loosening phenomenon occurs due to the insufficient pre-tensions in cables. From the calculated results, it can be discovered that the three cables can be loosening, but they can be loosening not at the same time. In other words, no restoring forces in the motion equations are vanished, so that the mast does not collapse. However, because of non-smoothness, this mast exhibits complex dynamical behavior. Hence, this mast may be damaged due to fatigue. 


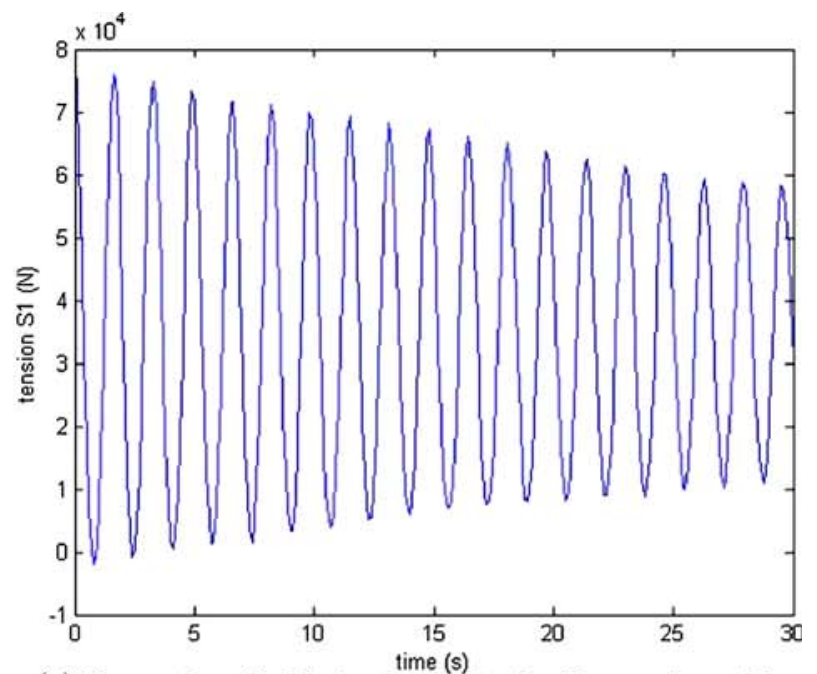

(a) The tension of cable 1 calculated using Petersen's model

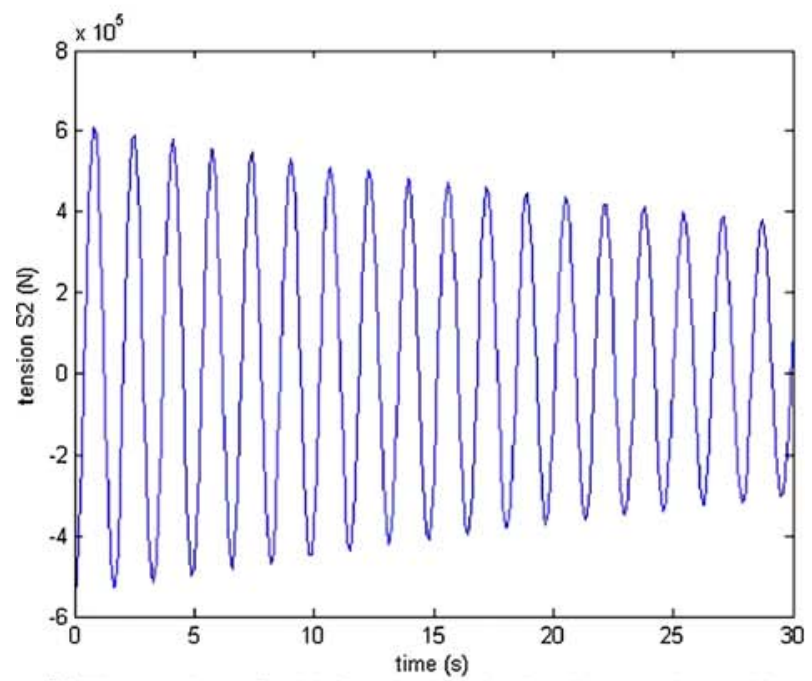

(b) The tension of cable 2 calculated using Petersen's model

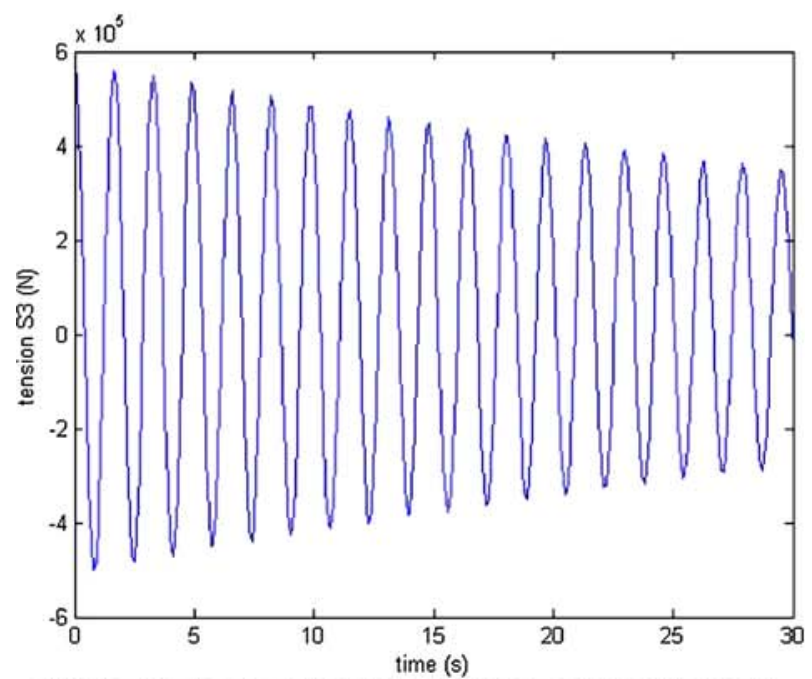

(c) The tension of cable 3 calculated using Petersen's model

Fig. 7 The results calculated using Petersen's model. a The tension of cable 1 calculated using Petersen's model. b The tension of cable 2 calculated using Petersen's model. c The tension of cable 3 calculated using Petersen's model 


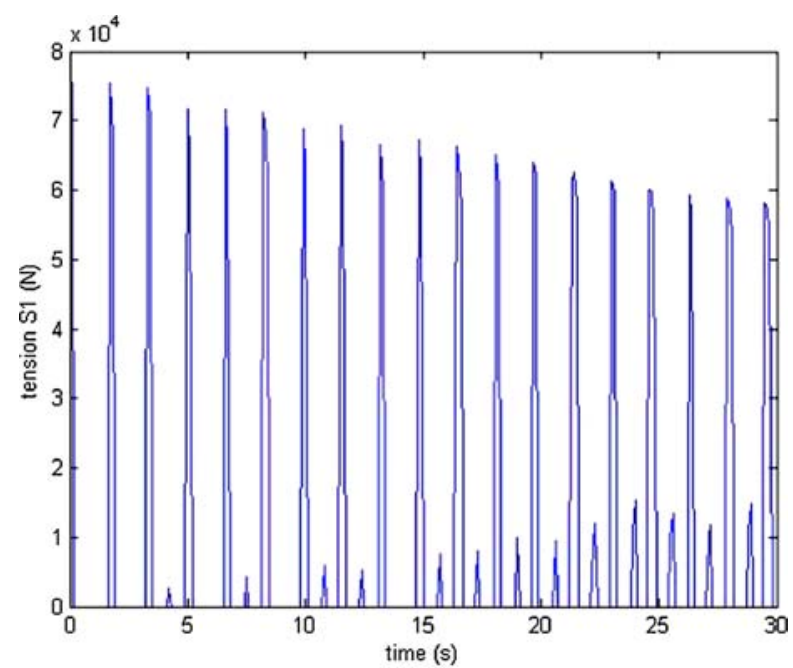

(a) The tension of cable 1 calculated using new model

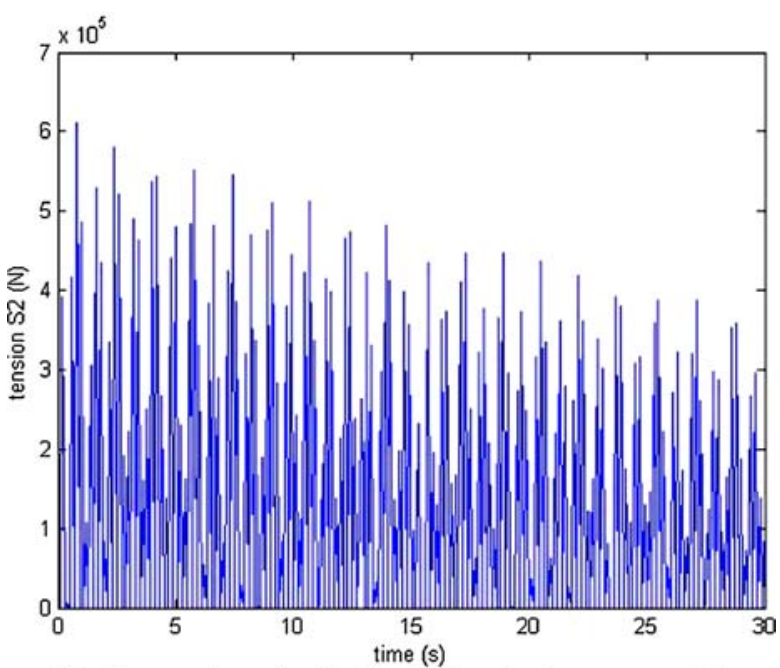

(b) The tension of cable 2 calculated using new model

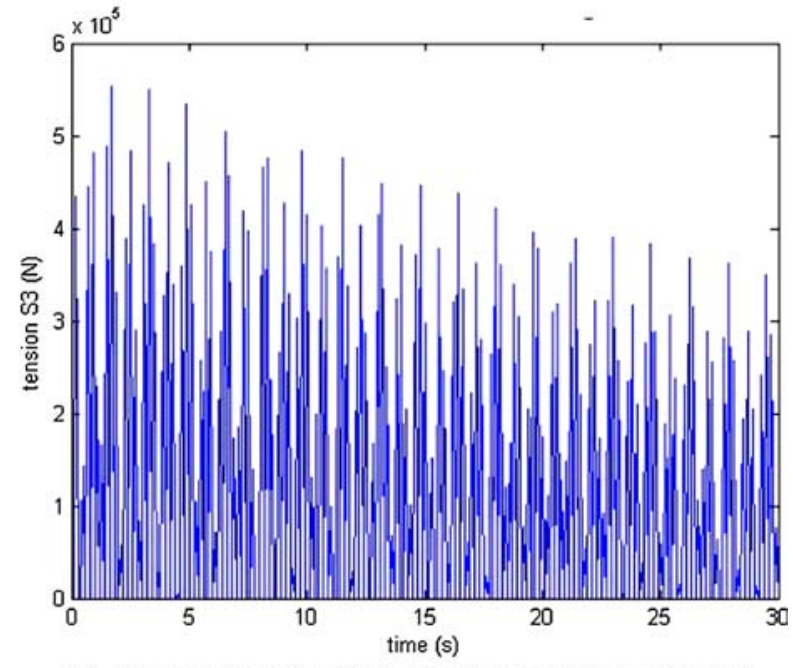

(c) The tension of cable 3 calculated using new model

Fig. 8 The calculated results using new model. a The tension of cable 1 calculated using new model. b The tension of cable 2 calculated using new model. $\mathbf{c}$ The tension of cable 3 calculated using new model 


\section{Conclusions}

Through the analysis of two examples, the following conclusions may be drawn:

(1) The loosening phenomenon of some cables may occur when the design of structures with cables is not reasonable.

(2) In modeling of cable-structures, consideration of the non-smooth behavior of those systems has been found to be necessary.

(3) The artificial NNWs used to analyze the dynamic systems with unilateral constraints may be a reliable and suitable method, which can save a large of time on computer.

\section{References}

1. Greenberg, D.P.: Inelastic analysis of suspension roof-structures. J Struct Div 96, 905-930 (1970)

2. Jonatowski, J.J., Birnstiel, C.: Inelastic stiffened suspension space structures. J Struct Div 96,1143-1166 (1970)

3. Murray, T.M., Willems, N.: Analysis of ineladtic suspension structures. J Struct Div 97,2791-2806 (1971)

4. Tene, Y., Epstein, M.: Nonlinear space trusses with tension-only members. In: International association for shell structures pacific symposium on tension structures and space frames, Tokyo, Japan and Kyoto, Japan (1971)

5. Jawerth, D., Schulz, H.: Ein Beitrag zur Frage der Eigenschwingungen, windanfachenden Kraefte und aerodynamischen Stabilitaet bei haengenden Daechern, Der Stahlbau, vol. 35(H.1), pp 1-8 (1966)

6. Maier, G., Asce, M., Contro, R.: Energy approach inelastic cable-structure analysis. J Eng Mech Div EM5, 531-548 (1975)

7. Panagiotopoulos, P.D.: A variational inequality approach to the inelastic stress-unilateral analysis of cable-structures. Comput Struct 6, 133-139 (1976)

8. Brogliato, B.: Nonsmooth mechanics. Springer, London (1999)

9. Monteiro Marques, M.D.P.: Differential inclusions in nonsmooth mechanical problems. Basel: Birkhaeuser 1993

10. Moreau, J.J.: Application of convex analysis to some problems of dry friction. In: Tends in application of pure mathematics to mechanics, London vol. 2 (1979)

11. Panagiotopoulos, P.D.: Inequality problems in mechanics and applications. Boston, Basel, Stuttgart: Birkaeuser 1985

12. Rockafellar, R.T.: Convex analysis. Princeton: Princeton University Press 1972

13. Panagiotopoulos, P.D.: Hemivariational inequalities. Berlin Heidelberg New York Springer, 1993

14. Murty, K.G.: Linear complementarity, linear and nonlinear programming. In: White, D.J. (ed.) Sigma series in applied mathematics. Berlin: Heldermann Verlag 1988

15. Ten Dam, A.A.: Representations of dynamical systems described by behavioral inequalities. In: Europen control conference, Groningen, NL, pp 1780-1783 (1993)

16. Ten Dam, A.A., Dnarshuis, E., Willems, J.C.: The contact problem for linear continuous-time dynamical systems: a geometric approach. IEEE Trans Autom Control 42(4), 458-472 (1993)

17. Van der Schaft, A., Schnacher, J.M.: The complementarity-slackness class of hybrid systems. Math Control Signal Syst 9 266-301 (1996)

18. Van der Schaft, A., Schnacher, J.M.: Complementarity modeling of hybrid systems. IEEE Trans Autom Control 43(4), 483-490 (1998) Special issue on hybrid systems

19. Petersen, Chr.: Chaotische Taumelschwingungen abgespannter Master. Der Stahlbau 61(H.6), 179-184 (1992)

20. Pfeiffer, F., Glocker, Chr.: Multibody dynamics with unilateral contacts. In: Nonlinear dynamics. New York: Wiley, 1996

21. Li-zhi, L., Hou-duo, Q.: A neural network for the linear complementarity problem. Math Comput Model 29, 9-18 (1999)

22. Bolotov, E.A.: On Gauss' principle, Izv. Fiz. Mat. Obshch. Pri Kazan University. Kazan: Kazan University Press 1916

23. Loetstedt, P.: Mechanical systems of rigid bodies subject to unilateral constraints. SIAM J Appl Math 42(2), 281-296 (1982)

24. Moreau, J.J.: Quadratic programming in mechanics: dynamics of one sided constraints. J SIAM Control 4(1), 153-158 (1966)

25. Sinitsyn, V.A.: The principle of least constraint for systems with non-restoring constraints. J Appl Math Mech 54(6), 920-925 (1990)

26. Baraff, D.: Issues in computing contact force for non-penetrating rigid bodies. Algorithmica 10, 292-352 (1993)

27. Hagan, M.T., Demuth, H.B., Beale, M.: Neural network design. Boston: PWS Publishing Compny 1996

28. Hopfield, J.J.: Neural networks and physical system with emergent collective computational properties. Proc Natl Acad Sci 79, 2554-2558 (1982)

29. Hopfield, J.J.: Neurons with graded response have collective computational properties like those of two-state neurons. Proc Natl Acad Sci 81, 3088-3092 (1984)

30. Hopfield, J.J., Tank, D.W.: Neural' computation of decisions in optimization problems. Biol Cybern 52, 141-154 (1985)

31. Tank, D.W., Hopfield, J.J.: Simple 'neural' optimization networks: an A/D converter, signal decision circuit and a linear programming circuit. IEEE Trans Circuits Syst 33(5), 533-541 (1986) 